\title{
ON THE DISTANCE BETWEEN ZEROES
}

\author{
WILLIAM T. PATULA
}

ABSTRACT. For the equation $x^{\prime \prime}+q(t) x=0$, let $x(t)$ be a solution with consecutive zeroes at $t=a$ and $t=b$. A simple inequality is proven that relates not only $a$ and $b$ to the integral of $q^{+}(t)$ but also any point $c \in(a, b)$ where $|x(t)|$ is maximized. As a corollary, it is shown that if the above equation is oscillatory and if $q^{+}(t) \epsilon$ $L^{p}[0, \infty), 1 \leq p<\infty$, then the distance between consecutive zeroes must become unbounded.

Consider the following second order linear differential equation:

$$
x^{\prime \prime}(t)+q(t) x(t)=0
$$

where $q(t)$ is continuous on some appropriate $t$ interval. Let $q^{+}(t) \equiv$ $\max [q(t), 0]$. Pertaining to (1), the following theorem of Hartman [3, p. 345] is known.

Theorem 1. Let $q(t)$ be real-valued and continuous for $a \leq t \leq b$. If $x(t)$ is a solution of (1) with two zeroes in $[a, b]$, then

$$
\int_{a}^{b}(t-a)(b-t) q^{+}(t) d t>(b-a) .
$$

Since $(b-a)^{2} / 4 \geq(t-a)(b-t)$ for $t \in(a, b)$, equation $(2) \Rightarrow$ that

$$
\frac{(b-a)^{2}}{4} \int_{a}^{b} q^{+}(t) d t>(b-a),
$$

or

$$
\int_{a}^{b} q^{+}(t) d t>\frac{4}{b-a}
$$

Thus Theorem 1 has as a corollary the following condition of Lyapunov. Again, see Hartman [3, p. 345].

Corollary 1. A necessary condition for any solution $x(t)$ of (1) to have two zeroes in $[a, b]$ is that $\int_{a}^{b} q^{+}(t) d t>4 /(b-a)$.

The lemma that we would like to present is the following.

Lemma 1。 Let $x(t)$ be a solution of (1), where $x(a)=x(b)=0$, and

Received by the editors July 2, 1974.

AMS (MOS) subject classifications (1970). Primary 34C10; Secondary 34B20.

Copyright $\odot 1975$, American Mathematical Society 
$x(t) \neq 0, t \in(a, b)$. Let $c$ be a point in $(a, b)$ where $|x(t)|$ is maximized. Then

(i) $\int_{a}^{c} q^{+}(t) d t>1 /(c-a)$,

(ii) $\int_{c}^{b} q^{+}(t) d t>1 /(b-c)$,

(iii) $\int_{a}^{b} q^{+}(t) d t>(b-a) /[(b-c)(c-a)]$.

Proof. Integrating (1) yields

$$
x^{\prime}(t)-x^{\prime}(c)=\int_{c}^{t} q^{-}(s) x(s) d s-\int_{c}^{t} q^{+}(s) x(s) d s .
$$

Note that $x^{\prime}(c)=0$. Another integration gives

$$
x(t)-x(c)=\int_{c}^{t}(t-s) q^{-}(s) x(s) d s-\int_{c}^{t}(t-s) q^{+}(s) x(s) d s .
$$

Let $t=b$, so that $x(b)=0$. Equation (5) implies that

$$
x(b)-x(c)=\int_{c}^{b}(b-s) q^{-}(s) x(s) d s-\int_{c}^{b}(b-s) q^{+}(s) x(s) d s
$$

or

$$
x(c)+\int_{c}^{b}(b-s) q^{-}(s) x(s) d s=\int_{c}^{b}(b-s) q^{+}(s) x(s) d s .
$$

W.L.O.G., we may assume $x(t) \geq 0, t \in[a, b]$. Thus we have

$$
\begin{aligned}
x(c) & \leq \int_{c}^{b}(b-s) q^{+}(s) x(s) d s<(b-c) \int_{c}^{b} q^{+}(s) x(s) d s \\
& \Rightarrow 1<(b-c) \int_{c}^{b} q^{+}(s) d s, \quad \text { since } x(s) \leq x(c), \text { if } s \in[a, b] \\
& \Rightarrow \int_{c}^{b} q^{+}(t) d t>\frac{1}{b-c} .
\end{aligned}
$$

This proves part (ii). Part (i) follows in a similar fashion, except that in equation (5), one now replaces $t$ by $a$. The sum of (i) and (ii) yields part (iii), which completes the lemma.

One way to view Lemma 1 is that it imposes some restrictions on the location of the point $c$ and thus the maximum of $|x(t)|$ in $[a, b]$. That is, $\int_{a}^{b} q^{+}(t) d t$ is a finite number. But

$$
\lim _{c \rightarrow a^{+}} \frac{b-a}{(b-c)(c-a)}=\lim _{c \rightarrow b^{-}} \frac{b-a}{(b-c)(c-a)}=\infty .
$$

Thus $c$ cannot be "too close" to $a$ or $b$. Also, it is interesting to note that $(b-a) /[(b-c)(c-a)] \geq 4 /(b-a)$. This means that under the hypothe- 
ses of Lemma 1, Corollary 1 follows from Lemma 1.

As a consequence of Lemma 1 (also Theorem 1 or Corollary 1), we have

Theorem 2. Suppose $q^{+}(t) \in L^{p}[0, \infty), 1 \leq p<\infty$. If (1) is oscillatory and if $x(t)$ is any solution, then the distance between consecutive zeroes of $x(t)$ must become infinite.

Proof. Suppose not. Then there exists a solution $x(t)$ with its sequence of zeroes $\left\{t_{n}\right\}$, which sequence has a subsequence $\left\{t_{n_{k}}\right\}$ such that $\left|t_{n_{k}+1}-t_{n_{k}}\right| \leq M<\infty \forall k$. Let $s_{n_{k}}$ be a point in $\left(t_{n_{k^{\prime}}} t_{n_{k}+1}\right)$ where $|x(t)|$ is maximized. Then $\left|s_{n_{k}}-t_{n_{k}}\right|<M$, for all $k$. Since $q^{+}(t) \in L^{p}[0, \infty), 1$ $\leq p<\infty$, choose $k$ so large that

$$
\left(\int_{t_{n_{k}}}^{\infty} q^{+}(t)^{p} d t\right)^{1 / p} \leq M^{-1-1 / r}, \quad \text { where } \frac{1}{p}+\frac{1}{r}=1 .
$$

From Lemma 1, part (i), we have

Thus

$$
\int_{t_{n_{k}}}^{s} q^{+}(t) d t>\frac{1}{s_{n_{k}}-t_{n_{k}}}
$$

$$
\begin{aligned}
1 & <\left(s_{n_{k}}-t_{n_{k}}\right) \int_{t_{n_{k}}}^{s} q_{k}^{+}(t) d t \\
& <\left(s_{n_{k}}-t_{n_{k}}\right)\left(\int_{t_{n_{k}}}^{s} q_{k}^{+}(t)^{p} d t\right)^{1 / p}\left(s_{n_{k}}-t_{n_{k}}\right)^{1 / r} \\
& <\left(s_{n_{k}}-t_{n_{k}}\right)^{1+1 / r}\left(\int_{t_{n_{k}}}^{\infty} q^{+}(t)^{p} d t\right)^{1 / p} \\
& <M^{1+1 / r} \cdot M^{-1-1 / r} \Rightarrow 1<1
\end{aligned}
$$

a contradiction. This completes the theorem.

Pertaining to (1), there is the following oscillation theorem of Wintner $[5]$.

If $\lim _{t \rightarrow \infty} \int_{0}^{t} q(s) d s=\infty$, then (1) is oscillatory.

The above condition enables us to construct some simple examples. Consider the equation

$$
x^{\prime \prime}+(1+t)^{-1} x=0, \quad t \geq 0 .
$$

The Wintner condition guarantees that $(6)$ is oscillatory. Since $1 /(1+t)$ $\epsilon L^{2}[0, \infty)$, Theorem 2 asserts that the distance between zeroes of any solution must become unbounded.

As another example, let 


$$
\begin{aligned}
q(t) & =1 /(n+1), \quad n+1 / n^{2} \leq t \leq(n+1)-1 /(n+1)^{2}, \quad n \geq 2 ; \\
& =(n)^{1 / 4}, \quad t=n, \quad n \geq 2 ;
\end{aligned}
$$

$=$ the line segment joining $\left(n-1 / n^{2}, 1 / n\right)$ to $\left(n, n^{1 / 4}\right)$

$$
\text { for } n-1 / n^{2} \leq t \leq n, \quad n \geq 2
$$

$=$ the line segment joining $\left(n, n^{1 / 4}\right)$ to $\left(n+1 / n^{2}, 1 /(n+1)\right)$

$$
\text { for } n \leq t \leq n+1 / n^{2}, \quad n \geq 2 \text {; }
$$

$=1 / 2$ for $0 \leq t \leq 7 / 4$.

So $q(t)$ has the following appearance.

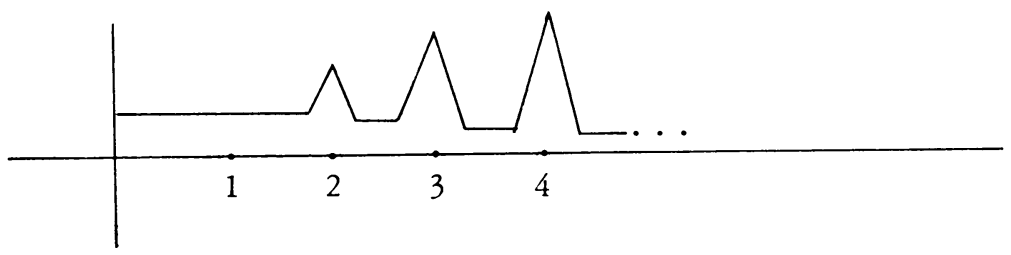

It is easy to verify that $\int_{0}^{\infty} q(t) d t=\infty$, but $\int_{0}^{\infty} q(t)^{2} d t<\infty$. The Wintner condition again implies that (1) is oscillatory, while Theorem 2 implies that the distance between zeroes is unbounded.

Theorem 2 can also be used to derive a known limit point result ( $\mathrm{Patula}$ and Wong $[4$, p. 10 , Corollary $])$. Note that for $t \geq 0$, equation (1) is called limit point, L.P., if at least one solution $x(t) \notin L^{2}[0, \infty)$. If any two linearly independent (and thus all) solutions are square integrable, (1) is called limit circle, L.C. See Coddington and Levinson [1, p. 225].

The following lemma is known (Patula and Wong [4, p. 11]).

Lemma 2. If equation (1) is L.C., then (1) is oscillatory, and the distance between consecutive zeroes of any solution tends to zero, as $t \rightarrow \infty$.

We can now prove the following limit point result.

Corollary 2. If $q^{+}(t) \in L^{p}[0, \infty), 1 \leq p<\infty$, then (1) is in the limit point classification.

Proof. Suppose not. Then (1) is L.C. Let $x(t)$ be any solution of (1). By Lemma 2, $x(t)$ oscillates and the distance between consecutive zeroes of any solution tends to zero, as $t \rightarrow \infty$. However, Theorem 2 maintains that if $x(t)$ oscillates, the distance between consecutive zeroes must become unbounded, a contradiction. Thus the equation must be limit point.

It should be noted that Theorem 2 does not hold for $p=\infty$, as evidenced by the simple example $x^{\prime \prime}+x=0$. However, it would be interesting to know 
if Theorem 2 is true for $0<p<1$. If it were, then Corollary 2 could also be extended to the case $0<p<1$. This would answer a question posed by Everitt, Giertz, and Weidmann [2, p. 346] as to whether or not (1) is limit point for $q^{+}(t) \in L^{p}[0, \infty), 0<p<1$.

Note added in proof. Lemma 1 is also contained in a paper by J. H. E. Cohn, Consecutive zeroes of solutions of ordinary second order differential equations, J. London Math. Soc. (2) 5 (1972), 465-468.

\section{REFERENCES}

1. E. A. Coddington and N. Levinson, Theory of ordinary differential equations,

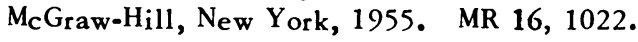

2. W. N. Everitt, M. Giertz and J. Weidmann, Some remarks on a separation and limit point criterion of second-order, ordinary differential equations, Math. Ann. 200 (1973), 335-346. $\# 1270$.

3. P. Hartman, Ordinary differential equations, Wiley, New York, 1964. MR 30

4. W. T. Patula and J. S. W. Wong, $A_{n} L^{p}$ analogue of the Weyl alternative, Math. Ann. 197, (1972), 9-28. MR $45 \# 8913$.

5. A. Wintner, A criterion of oscillatory stability, Quart. Appl. Math. 7 (1949), 115-117. MR 10, 456 .

DEPARTMENT OF MATHEMATICS, SOUTHERN ILLINOIS UNIVERSITY, CARBONDALE,
INOIS 62901 ILLINOIS 62901 